\title{
Q1 Performance analysis of orthogonal frequency division multiplexing systems in dispersive indoor power line channels inflicting asynchronous impulsive noise
}

\section{ISSN 1751-8628}

Received on 25th July 2015

Revised on 11th November 2015 Accepted on 5th December 2015 doi: 10.1049/iet-com.2015.0702 www.ietdl.org

\author{
Q2 Hongming Zhang, Lie-Liang Yang, Lajos Hanzo $\bowtie$ \\ 15 School of Electronics and Computer Science, University of Southampton, SO17 1BJ, UK \\ 凶E-mail:Ih@ecs.soton.ac.uk
}

\begin{abstract}
Hidden semi-Markov modelling of the asynchronous impulsive noise (IN) encountered in indoor broadband power line communications (PLCs) is investigated by considering the statistical distributions of both the inter-arrival time and the duration of asynchronous IN components. Then, the bit error ratio (BER) of orthogonal frequency division multiplexing systems using $Q$-ary quadrature amplitude modulation is analysed with the aid of the proposed noise model, when communicating over dispersive indoor power line channels inflicting asynchronous IN in addition to the background noise. The authors' simulation results confirm the accuracy of the analysis and quantify the impact of various factors on the achievable BER performance. The grave impact of asynchronous IN on indoor broadband PLCs suggests that efficient techniques have to be designed for mitigating its effects.
\end{abstract}

\section{Introduction}

Power line communication (PLC) is one of attractive candidates for the so-called 'last mile' communications due to its cost efficiency, since it exploits the existing grid structure [1]. However, since the electrical supply networks have not been designed for data transmissions, they constitute a hostile propagation environment

35 [2], where the multipath-induced dispersion and the impulsive noise (IN) are the two fundamental impediments in the way of high-integrity communications. Nonetheless, multicarrier comm unication techniques are capable of mitigating the multipath effects in PLC, whilst spreading the effects of IN over all subcarriers [3].

40 A range of advanced techniques were also reported in [4-6].

As a further impairment, the attenuation encountered in PLC is the result of the skin effect and dielectric losses [7]. By contrast, dispersive multipath signal propagation in PLC is caused by the mismatch of the impedance between a transmitter and its corresponding receiver [8]. As a result, a transmitted symbol may

45 be spread over several adjacent symbols at the receiver, imposing inter-symbol interference (ISI), which was investigated in [8-10]. Furthermore, the statistical characteristics of ISI-contaminated power line channels were studied in [11-15].

In PLC, the noise can usually be classified into two categories: 50 background noise and IN $[16,17]$. The $\mathrm{IN}$ is typically characterised by the duration, inter-arrival time and power of its components [16]. According to its behaviour with respect to the mains cycle, IN can be classified into three types, namely the periodic mains-synchronous $\mathrm{IN}$, the periodic $\mathrm{IN}$ that is asynchronous with the mains, as well as the asynchronous IN [16],

55 which is mainly caused by the connection and disconnection of electrical devices. Typically, the asynchronous IN is the most dominant impairment of broadband PLCs due to its high power and unpredictable nature. Therefore, we focus our attention on the asynchronous IN in this treatise. Several studies demonstrated [16,

$6018,19]$ that the average duration of IN bursts in PLC is relatively long in comparison to the IN bursts of wireless communications. More specifically, the measurement results of [16] showed that the average duration of the asynchronous IN bursts in PLC varies between tens of microseconds and tens of milliseconds. By contrast, in wireless communications, the duration of IN bursts is if signal samples with a symbol-duration of say $10 \mathrm{~ns}$ are transmitted at a Nyquist-rate of 100 MBaud, then more than $10^{3}$ successive symbols may be corrupted by an IN burst, once it occurs. By contrast, in wireless communications, no more than ten successive samples are impaired by an IN burst. Naturally, these long impulsive bursts may inflict bursts of errors. As a result, the system's performance may be severely degraded, especially in high data-rate transmissions relying on short symbol durations. In the literature, two special cases of the multi-component Gaussian mixture model [21] have been used for modelling the IN, which are respectively referred to as the Bernoulli-Gaussian model [22] and the Middleton Class A model [23]. Specifically, in [3], the classic Poisson process was employed for evaluating the probability of IN occurrences, where the Bernoulli-Gaussian model was assumed. In [24], Markov chains were introduced for calculating the so-called impulsive index of the Middleton Class A model. However, in the above-mentioned pair of models the IN samples were independently generated, which fails to reflect the typical bursty behaviour of IN in PLC.

To mimic the bursty behaviour of IN, Markov chains were employed in [16, 25, 26]. In [16], a so-called partitioned Markov chain, which considered a set of $\mathrm{IN}-$ free states and a set of impulsive states were employed for modelling the bursty behaviour of IN. Later in [25], a two-state Markov chain model was adopted for mimicking the bursty nature of $\mathrm{IN}$. In this model, the noise samples were generated as white Gaussian noise, where noise samples having an increased variance were generated to represent IN. Recently, a more accurate four-state Markov chain model was proposed [26], where the IN samples were assumed to obey the Middleton Class A model of [23]. Although these Markov chain-based noise models succeeded in generating bursty IN, the statistical accuracy of the state durations was not carefully considered.

Against this background, our new contribution is that the hidden semi-Markov model (HSMM) [27], which was shown to accurately model the duration of burst events in [28], is applied for modelling the bursty behaviour of asynchronous IN, when analytically characterising the bit error ratio (BER) performance of orthogonal frequency division multiplexing (OFDM)-based PLC systems using $Q$-ary quadrature amplitude modulation (QAM). Our simulation results verify the accuracy of our analytical results. 
The rest of the paper is organised as follows. In Section 2, we describe the system and introduce our noise model. Section 3

\section{System and noise modelling}

\subsection{OFDM signalling}

We consider a discrete-time baseband equivalent model of the OFDM system, which is illustrated in Fig. 1. In the OFDM system, a block of $Q$-ary QAM symbols is serial-to-parallel converted and then the parallel symbols $\boldsymbol{X}=\left[X_{0}, X_{1}, \ldots, X_{M-1}\right]^{\mathrm{T}}$ are modulated with the aid of inverse fast Fourier transform (IFFT), yielding the time-domain (TD) signals $\boldsymbol{x}=\left[x_{0}, x_{1}, \ldots, x_{M-1}\right]^{\mathrm{T}}$, which can be expressed as

$$
\boldsymbol{x}=\mathcal{F}^{\mathrm{H}} \boldsymbol{X}
$$

where $\mathcal{F}$ is the normalised discrete Fourier transform (DFT) matrix [29] satisfying $\mathcal{F F}^{\mathrm{H}}=\mathcal{F}^{\mathrm{H}} \mathcal{F}=\boldsymbol{I}_{M}$. Hence, $\mathcal{F}$ is an orthonormal matrix. After concatenating the cyclic prefix (CP), the TD transmitted symbols are arranged in the form of

$$
\tilde{\boldsymbol{x}}=\left[x_{M-L^{\prime}}, x_{M-L^{\prime}+1}, \ldots, x_{M-1}, x_{0}, x_{1}, \ldots, x_{M-1}\right]^{\mathrm{T}},
$$

where $L^{\prime}$ is the length of the CP.

In OFDM systems, the bandwidth of each subchannel is usually significantly smaller than the coherence bandwidth of the communication channel. Hence, each of the subcarriers experiences flat fading. Therefore, after removing the $\mathrm{CP}$ at the receiver, the received baseband equivalent observations $\boldsymbol{y}=\left[y_{0}, y_{1}\right.$, $\left.\ldots, y_{M-1}\right]^{\mathrm{T}}$ can be formulated as

$$
\boldsymbol{y}=\widetilde{\boldsymbol{H}} \boldsymbol{x}+\boldsymbol{n},
$$

where $\widetilde{\boldsymbol{H}}$ is an $(M \times M)$-element circulant matrix, which can be diagonalised by the DFT matrix, giving $\widetilde{\boldsymbol{H}}=\mathcal{F}^{\mathrm{H}} \boldsymbol{H} \mathcal{F}$, where $\boldsymbol{H}$ is a diagonal matrix. In (3), $\boldsymbol{n}$ is the noise vector, which includes both the Gaussian background noise and the IN, as it will be detailed in Section 2.3.

Assuming that perfect synchronisation has been achieved at the receiver, the decision variables can be obtained with the aid of the fast Fourier transform (FFT) operation as

$$
\begin{aligned}
\widetilde{\boldsymbol{X}} & =\mathcal{F} \boldsymbol{y}=\mathcal{F}(\widetilde{\boldsymbol{H}} \boldsymbol{x}+\boldsymbol{n})=\mathcal{F}\left(\widetilde{\boldsymbol{H}} \mathcal{F}^{\mathrm{H}} \boldsymbol{X}+\boldsymbol{n}\right) \\
& =\mathcal{F} \mathcal{F}^{\mathrm{H}} \boldsymbol{H} \mathcal{F} \mathcal{F}^{\mathrm{H}} \boldsymbol{X}+\mathcal{F} \boldsymbol{n}=\boldsymbol{H} \boldsymbol{X}+\boldsymbol{N},
\end{aligned}
$$

where we have $\boldsymbol{N}=\mathcal{F} \boldsymbol{n}$. Furthermore, we assume that the phase rotations in $\boldsymbol{H}$ have been perfectly compensated by the coherent detection scheme. Then, based on (4), the transmitted symbols in $\boldsymbol{X}$ can be detected according to the decision rules of $Q$-ary QAM [30], where the detection performance is affected by both the channel attenuations in $\boldsymbol{H}$ and the noise samples in $\boldsymbol{N}$.

\subsection{Modelling of indoor power line channels}

Due to the frequent connection and disconnection of various types of loads, as well as the presence of cable branches, indoor PLC channels exhibit a time-variant frequency-selective channel transfer function (CTF). However, we can usually assume that the channel remains constant during a single OFDM symbol. This assumption is reasonable, since in high-speed data transmissions the PLC channels vary rather slowly. Furthermore, in contrast to wireless multipath channels, the multipath effect of PLC channels can be analytically calculated [9] with the aid of the CTF between any two outlets. According to [8], the CTF of the PLC channels can be expressed as

$$
H(f)=\sum_{i=0}^{L_{e}-1} g_{i} \mathrm{e}^{-\alpha(f) v_{p} \tau_{i}} \mathrm{e}^{-\mathrm{j} 2 \pi f \tau_{i}},
$$

where $L_{e}$ denotes the number of non-negligible paths; $\left|g_{i}\right|<1$ is the reflection factor, which is determined both by the number of discontinuities included in the $i$ th path, as well as by the reflection coefficient and the transmission coefficient of the $i$ th path [9]; $\alpha(f)$ is a frequency-dependent attenuation factor, which is related both to the dielectric losses and ohmic losses [18]; and finally, $\tau_{i}$ is the delay of the $i$ th reflected path, while $v_{p}$ is the phase velocity. Correspondingly, the channel impulse response (CIR) can be formulated as $h(t)=\mathcal{F}^{-1}\{H(f)\}$, where $\mathcal{F}^{-1}\{x\}$ denotes the inverse Fourier transform of $x$. Note that the channel model expressed in (5) uses a top-down approach for modelling the PLC channels, where the associated values of the parameters can be obtained from measurements [8].

Specifically, when the OFDM signals of (2) are transmitted over the PLC channel characterised by (5), and the received signals are sampled at intervals of $\Delta t=T_{\mathrm{S}} M$ representing the chip duration, with $T_{\mathrm{s}}$ being the OFDM symbol duration, the chip-sampled baseband equivalent CIR after filtering can be expressed as $\boldsymbol{h}=$ $\left[h_{0}, h_{1}, \ldots, h_{L-1}\right]^{\mathrm{T}}$, where $L \simeq \tau_{L_{e-1}} \Delta t$ is the discretised length of

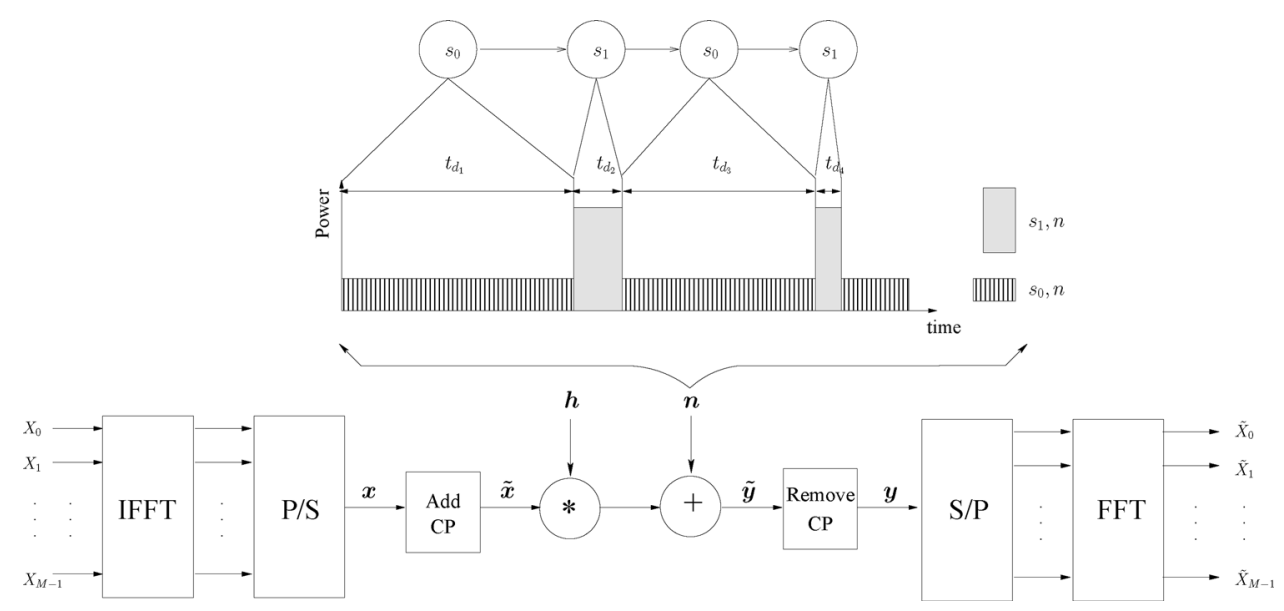

Fig. 1 System and noise model. $s_{0}$ : state without $I N ; s_{1}$ : state with $I N . \mathrm{t}_{\mathrm{d}}$ : duration time of state $s_{i}(\mathrm{i}=0,1)$ 
265 the delay spread, which is an integer multiple of the chip-duration. Upon carrying out the FFT, the fading gains of the $M$ subcarriers, which are denoted by $\boldsymbol{h}_{f}=\left[H_{0}, H_{1}, \ldots, H_{M-1}\right]^{\mathrm{T}}$, can be expressed as

$$
\boldsymbol{h}_{f}=\sqrt{M} \mathcal{F} \boldsymbol{h}_{M},
$$

270

where we have $\boldsymbol{h}_{M}=\left[\boldsymbol{h}^{\mathrm{T}}, \mathbf{0}_{1 \times(M-L)}\right]^{\mathrm{T}}$. Consequently, the diagonal matrix $\boldsymbol{H}$ seen in (4) is given by $\boldsymbol{H}=\operatorname{diag}\left\{H_{0}, H_{1}, \ldots, H_{M-1}\right\}$.

\subsection{Noise modelling}

The HSMM [27] is an extension of the classic hidden Markov model [31], where the underlying stochastic process obeys a semi-Markov chain, while the different states may have different durations [28]. Based on the HSMM, below we show a noise model for PLC.

280 As shown in Fig. 1, the noise contaminating the TD OFDM signals has two states, where $s_{0}$ represents the sole presence of the Gaussian background noise, while $s_{1}$ represents the presence of both the Gaussian background noise and IN. Explicitly, as shown in Fig. 1, the duration of state $s_{0}$ corresponds to that of the unperturbed interval between two adjacent IN bursts, which we hence refer to as 'inter-burst time'. By contrast, the duration of state $s_{1}$ corresponds to that of an IN burst. Again, the IN considered in this paper is assumed to be asynchronous IN. As the measurement results of [16] demonstrated, both the burst-duration and the unperturbed inter-burst time obey negative exponential

290 distributions. Furthermore, the burst-duration of asynchronous IN is much lower than the inter-burst time. Since the exponential distributions are memoryless [32], the probability density functions (PDFs) of the state-durations $t_{d}$ conditioned on $s_{0}$ and $s_{1}$, respectively, can be expressed as

$$
f\left(t_{d} \mid s_{i}\right)=\frac{1}{\Omega_{s_{i}, t_{d}}} \exp \left(-\frac{t_{d}}{\Omega_{s_{i}, t_{d}}}\right), \quad i=0,1,
$$

300

where $\Omega_{s_{i}, t_{d}}=E\left[t_{d} \mid s_{i}\right]$ denotes the conditional expectations of $t_{d}$, given $s_{i}$ for $i=0,1$. In addition, we define the ratio of the average burst-duration to the average inter-burst duration (ADIR) as $\Lambda=\Omega_{s_{1}, t_{d}} \Omega_{s_{0}, t_{d}}$.

Let us assume perfect receiver synchronisation and that the received samples arrive at the time instants of $0, \Delta t, 2 \Delta t, \ldots$,

$305(M-1) \Delta t$. Let us define the normalised HSMM state-duration as $d=\left[t_{d} \Delta t\right]$, where $[x]$ represents the rounded integer closest to the real value $x$. Then, the mean of the normalised HSMM state-duration $d$ conditioned on $s_{0}$ and $s_{1}$ can be calculated as $\Omega_{s_{0}, d}=\Omega_{s_{0}, t_{d}} \Delta t$ and $\Omega_{s_{1}, d}=\Omega_{s_{1}, t_{d}} \Delta t$, respectively. According to Appendix, the probability mass functions (PMFs) of $d$ conditioned on $s_{0}$ and $s_{1}$ are given by

$$
p\left(d \mid s_{i}\right)=G\left(d, \Omega_{s_{i}, d}\right), \quad i=0,1,
$$

for $d=0,1, \ldots$, where the function $G(x, \Omega)$ is defined in (47).

Let us assume that the initial state of the noise process is chosen from $\left\{s_{0}, s_{1}\right\}$ with equal probability of 0.5 . Then, according to the characteristics of the noise process, as shown in Fig. 1, the states $s_{0}$ and $s_{1}$ occur alternatively. Hence, the self-transition probabilities of state $s_{0}$ and state $s_{1}$ are 0 , i.e. we have $P_{00}=P_{11}=0$, where $P_{i j}$ denotes the transition probability from state $s_{i}$ to state $s_{j}$. Therefore, we have the transition probabilities of $P_{01}=P_{10}=1$. Moreover, when assuming that the noise samples during both states obey the complex-valued Gaussian distributions with a mean of zero and with their individual variances depending on the corresponding state, the PDFs of a noise sample $n$ conditioned on $s_{0}$ and $s_{1}$ can be expressed as

$$
f\left(n \mid s_{i}\right)=\frac{1}{2 \pi \sigma_{s_{i}, n}^{2}} \exp \left(-\frac{|n|^{2}}{2 \sigma_{s_{i}, n}^{2}}\right), \quad i=0,1,
$$

where $\sigma_{s_{i}, n}^{2}$ denotes the noise variances in state $s_{i}$ for $i=0,1$. In addition, for convenience, we define the ratio of these variances as $\mu=\sigma_{s_{1}, n}^{2} \sigma_{s_{0}, n}^{2}$.

Above, the proposed noise model has been described in the TD. However, as shown in (4), the detection performance of the OFDM system is affected by the noise samples in the frequency-domain (FD), which are given by $\boldsymbol{N}=\mathcal{F} \boldsymbol{n}$. Therefore, in the following section, we analyse the statistics of the noise in the FD.

\section{Statistics of FD noise samples}

Recall from our previous discussions that the OFDM symbols are impaired by the noise samples of the state sequence $\hat{\boldsymbol{s}}=\left\{\hat{s}_{1}, \hat{s}_{2}, \ldots, \hat{s}_{T}\right\}$, where $\hat{s}_{j} \in\left\{s_{0}, s_{1}\right\}, T$ denotes the number of noise states encountered during a transmission block and any two consecutive states of $\hat{\boldsymbol{s}}$ satisfy $\hat{s}_{j} \neq \hat{s}_{j+1}$. Then, as shown in Fig. 2, a TD OFDM symbol may be corrupted by one of three different types of noise. As shown in Fig. $2 a$, the first one is when the $M$ noise samples are all from the same state, either $s_{0}$ or $s_{1}$. During the second type the noise samples are from a pair of different states $\hat{s}_{j}$ and $\hat{s}_{j+1}$, namely from $s_{0}$ and $s_{1}$, as shown in Fig. $2 b$. Finally, during the third type the noise samples are from more than two successive states $\hat{s}_{j}, \hat{s}_{j+1}, \ldots, \hat{s}_{j+k}$, where $k \geq 2$, as exemplified in Fig. 2c. However, in practice, the average inter-arrival time of impulsive bursts is usually much higher than the OFDM symbol duration, i.e. we have $\Omega_{s_{0}, t_{d}} \gg T_{\mathrm{s}}$. Hence, for the third type we may only consider the case of $k=2$ and the scenario of having three successive states $s_{0}, s_{1}, s_{0}$.

It can be readily shown that in the FD there are also two states, namely $S_{0}$ without IN and $S_{1}$ representing the presence of IN. Let us define the duration of state $S_{0}$ in terms of the number of successive OFDM symbols that are not corrupted by IN, while the duration of state $S_{1}$ as the number of successive OFDM symbols impaired by IN. For convenience of analysis, we let $\eta$ be the number of TD chips spanning from the start of an OFDM symbol to the time instant of a state transition, as shown in Fig. 3. Since $\eta$ is the offset relative to the start of an OFDM symbol, we can assume that $\eta$ obeys the discrete uniform distribution having a PMF given by

$$
p(\eta)=\frac{1}{M}, \quad \eta=0,1, \ldots, M-1
$$

On the basis of the above assumptions, for a given FFT size of $M$, we can show that when the noise samples in $\boldsymbol{n}$ are generated during state
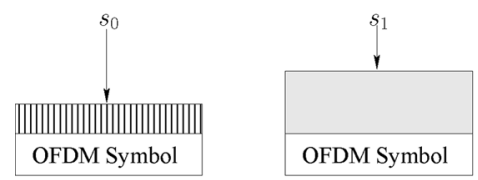

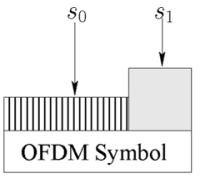

$b$
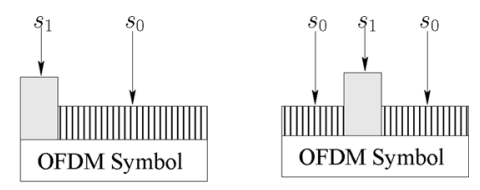

Fig. 2 Stylised illustration of noise types in the TD 

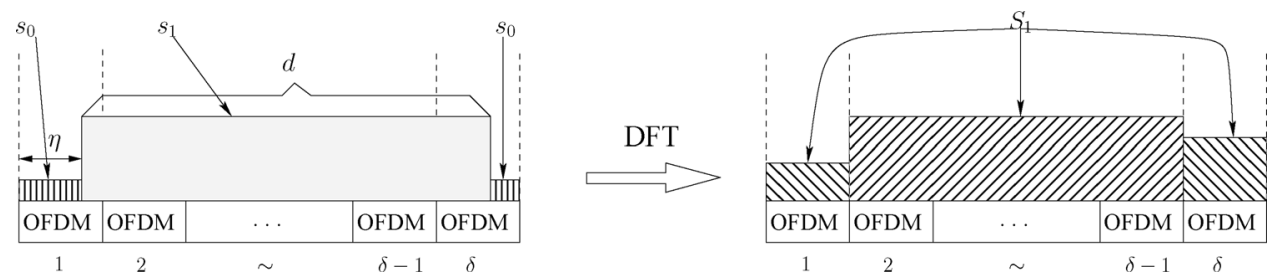

Fig. 3 Illustration of $\delta$ successive OFDM symbols, which are corrupted by noise samples in both the TD and in the corresponding FD

$s_{i}$, where $i \in\{0,1\}$, with a normalised HSMM state duration of $d$, the corresponding observations in the FD belong to state $S_{i}$ with a 410 duration of $\delta$, where for $i \in\{0,1\}, \delta$ is given by

$$
\delta= \begin{cases}q-2+2 i & \text { if } 1-i \leq \eta \leq r-1+i \\ q-1+2 i & \text { if } r+i \leq \eta \leq M-1 \\ & \text { or } \eta=0 \text { for } i=0\end{cases}
$$

with $q=\lceil d M\rceil$ as well as $r=\lceil d M\rceil M-d$ being positive integers and $\lceil x\rceil$ denotes the smallest integer larger than $x$. It should be noted that our assumption for the third type of noise in the TD guarantees that $\delta$ is always a positive integer.

Furthermore, as shown in Appendix, the PMFs of $\delta$ conditioned on $S_{i}$, where $i=0,1$, are given by

$$
\begin{aligned}
p\left(\delta \mid S_{i}\right)= & \Omega_{S_{i}, \delta}\left[G\left(\frac{\delta+(-1)^{i}}{2}, \Omega_{S_{i}, \delta}\right)\right]^{2} \\
& -\zeta\left(\Omega_{S_{i}, \delta}\right), \quad \delta=1,2, \ldots,
\end{aligned}
$$

where $\zeta\left(\Omega_{S_{i}}, \delta\right)$ is defined in $(50), \Omega_{S_{0}}, \delta=E\left(\delta \mid S_{0}\right)$ and $\Omega_{S_{1}}, \delta=E\left(\delta \mid S_{1}\right)$ denote the expectations of $\delta$, given $S_{0}$ and $S_{1}$, respectively.

Let the noise samples generated in state $S_{i}$ with a duration of $\delta$ be expressed as $\boldsymbol{N}=\{\boldsymbol{N}(1), \boldsymbol{N}(2), \ldots, \boldsymbol{N}(\delta)\}$, where $\boldsymbol{N}(j)=\mathcal{F}[\boldsymbol{n}(j)]$, and let the number of the samples in $\boldsymbol{n}(j)$ that are from the state $s_{1}$ be $\kappa$, where $\kappa \in\{0,1, \ldots, M\}$. As a result, there are $(M-\kappa)$ samples in $\boldsymbol{n}(j)$ generated from the state $s_{0}$. Then, we can show that the noise samples in $\boldsymbol{N}(j)$ are complex-valued Gaussian random variables with a mean of zero and a variance given by

$$
\begin{aligned}
\sigma_{S_{i}, N}^{2}(j) & =\frac{(M-\kappa) \sigma_{s_{0}, n}^{2}+\kappa \sigma_{s_{1}, n}^{2}}{M} \\
& =\left[1+\frac{\kappa(\mu-1)}{M}\right] \sigma_{s_{0}, n}^{2},
\end{aligned}
$$

where $\kappa$ is dependent not only on the state $S_{i}$ but also on the duration $\delta$, which are detailed below.

In the following analysis, we assume that each transmission is sufficiently long for ensuring that the statistical distributions of the durations of the FD states $S_{0}$ and $S_{1}$ can be described by the PMFs of (12). Furthermore, we can show that during each transmission, we have $\left|K_{0}-K_{1}\right|=1$, where the number of FD states $S_{i}$ is denoted by $K_{i}$. Based on our assumptions, $K_{0}$ and $K_{1}$ are sufficiently large so that $K_{0} K_{1} \simeq 1$. Let us denote the $k$ th duration conditioned on state $S_{i}$ as $\delta_{S_{i, k}}$, where we have $\sum_{k=1}^{K_{i}} \delta_{S_{i}, k}=\Omega_{S_{i}, \delta} K_{i}$ for $i=0$, 1. Hence, for a given FD observation $\tilde{\boldsymbol{X}}$, which represents the OFDM symbol of (4), the a posteriori probability of the OFDM symbol becoming impaired by noise samples generated by the state $S_{i}$ can be expressed as

$$
\begin{aligned}
P\left(S_{i} \mid \tilde{\boldsymbol{X}}\right) & =\frac{\sum_{k=1}^{K_{i}} \delta_{S_{i}, k}}{\sum_{k=1}^{K_{0}} \delta_{S_{0}, k}+\sum_{k=1}^{K_{1}} \delta_{S_{1}, k}}=\frac{\Omega_{S_{i}, \delta} K_{i}}{\Omega_{S_{0}, \delta} K_{0}+\Omega_{S_{1}, \delta} K_{1}} \\
& \simeq \frac{\Omega_{S_{i}, \delta}}{\Omega_{S_{0}, \delta}+\Omega_{S_{1}, \delta}} .
\end{aligned}
$$

Substituting (45) into (14) gives

$$
P\left(S_{i} \mid \tilde{\boldsymbol{X}}\right) \simeq \frac{\Lambda^{i}}{1+\Lambda}
$$

where $\Lambda$ was defined in Section 2.3. Then, according to Bayes' rule, we can show that

$$
\begin{aligned}
P\left(\delta, S_{i} \mid \tilde{\boldsymbol{X}}\right) & =\frac{P\left(\delta, S_{i}, \widetilde{\boldsymbol{X}}\right)}{P(\tilde{\boldsymbol{X}})} \\
& =\frac{P\left(\delta \mid S_{i}, \tilde{\boldsymbol{X}}\right) P\left(S_{i}, \tilde{\boldsymbol{X}}\right)}{P(\tilde{\boldsymbol{X}})} \\
& =P\left(\delta \mid S_{i}, \tilde{\boldsymbol{X}}\right) P\left(S_{i} \mid \tilde{\boldsymbol{X}}\right),
\end{aligned}
$$

where $P\left(\delta \mid S_{i}, \tilde{\boldsymbol{X}}\right)$ is the probability that for a given OFDM symbol impaired by the noise samples generated by the state $S_{i}$, this OFDM symbol belongs to that specific set of $\delta$ successive OFDM symbols, all of which are impaired by the noise samples generated by the state $S_{i}$, as shown in Fig. 3. Let the number of states $S_{i}$ having a duration of $\delta$ successive OFDM symbols in the FD be denoted as $k_{\delta}$, where we have $\sum_{\delta=1}^{\infty} k_{\delta}=K_{i}$. Furthermore, we have $p\left(\delta \mid S_{i}\right)=k_{\delta} K_{i}$. Consequently, it can be shown that

$$
\begin{aligned}
P\left(\delta \mid S_{i}, \tilde{\boldsymbol{X}}\right) & =\frac{\delta k_{\delta}}{\sum_{k=1}^{K_{i}} \delta_{k}}=\frac{\delta k_{\delta}}{\Omega_{S_{i}, \delta} K_{i}} \\
& =\frac{\delta p\left(\delta \mid S_{i}\right)}{\Omega_{S_{i}, \delta}}
\end{aligned}
$$

Upon substituting (15) and (17) into (16), we arrive at

$$
P\left(\delta, S_{i} \mid \widetilde{\boldsymbol{X}}\right)=\frac{\Lambda^{i}}{1+\Lambda} \frac{\delta p\left(\delta \mid S_{i}\right)}{\Omega_{S_{i}, \delta}}
$$

Below, we derive the PMF $p(\kappa)$, where $\kappa$ assumes different values.

\section{$3.1 \kappa=0$}

First, when only the FD state $S_{0}$ is encountered during the transmission of $\delta$ OFDM symbols, we have $\kappa=0$. Thus, the probability of $\kappa=0$ is equal to the probability of an OFDM symbol being impaired by the noise samples generated by $S_{0}$, which is given by

$$
P(\kappa)=P\left(S_{0} \mid \tilde{\boldsymbol{X}}\right) \simeq \frac{1}{1+\Lambda}, \quad \kappa=0
$$

\section{$3.2 \kappa=1,2, \ldots, M-1$}

Second, as shown in Fig. 3, if the FD state $S_{1}$ having a duration of 525 $\delta \geq 2$ occurs, we may have $1 \leq \kappa \leq M-1$ [Here, $\kappa$ maybe equal $\mathbf{Q 3}$ to $M$. However, recalling our assumptions that the transmission time is long enough and $M$ is sufficiently large, the case where 
state $S_{1}$ with a duration of $\delta \geq 2$ and $\kappa=M$ for $j=1$ occurs rarely. negligible.], when the first and the last ( $\delta$ th) OFDM symbols are considered. Therefore, we have

$$
\operatorname{Pr}_{1}(1 \leq \kappa \leq M-1)=\sum_{\delta=2}^{\infty} \frac{2}{\delta} P\left(\delta, S_{1} \mid \tilde{\boldsymbol{X}}\right),
$$

where $2 \delta$ is the probability of the first and the last ( $\delta$ th) OFDM symbols being picked from the $\delta$ consecutive OFDM symbols.

Furthermore, with the aid of (18) and (46a), we arrive at

$$
\begin{aligned}
\operatorname{Pr}_{1}(1 \leq \kappa \leq M-1) & =\frac{\Lambda}{1+\Lambda} \frac{2 \sum_{\delta=2}^{\infty} p\left(\delta \mid S_{1}\right)}{\Omega_{S_{1}, \delta}} \\
& =\frac{2 \Lambda}{1+\Lambda}\left[\frac{1}{\Omega_{S_{1}, \delta}}-1+G\left(0.5, \Omega_{S_{1}, \delta}\right)\right],
\end{aligned}
$$

550

where $G(x, \Omega)$ is defined in (47).

As shown in Fig. $2 b$, we have $\kappa=(M-\eta)$ and $\eta$ obeys the uniform distribution. Therefore, we have

$$
\begin{aligned}
P_{1}(\kappa) & =\frac{1}{M-1} \operatorname{Pr}_{1}(1 \leq \kappa \leq M-1) \\
& =\frac{\Lambda}{1+\Lambda} \frac{2}{M-1}\left[\frac{1}{\Omega_{S_{1}, \delta}}-1+G\left(0.5, \Omega_{S_{1}, \delta}\right)\right] \\
& =\frac{\Lambda}{1+\Lambda}\left[\frac{\left(2 / \Omega_{S_{1}, \delta}\right)+2 G\left(0.5, \Omega_{S_{1}, \delta}\right)-2}{(M-1)}\right]
\end{aligned}
$$

for $\kappa=1,2, \ldots, M-1$. Equation (22) shows that $P_{1}(\kappa)$ is independent of $\kappa$

On the other hand, when the FD state $S_{1}$ with a duration of $\delta=1$ occurs, we may also have $1 \leq \kappa \leq M-1$. In this case, as shown in Fig. $2 c$, we have $\kappa=d$. Thus, by substituting (43a) into (18), we can show that

570

575

In this case, we find that the distribution of $\kappa$ obeys the PMF $G\left(\kappa, \Omega_{s_{1, d}}\right)$. Thus, we can write

580

$$
P_{2}(\kappa)=\frac{\Lambda}{1+\Lambda} \frac{(M-\kappa+1) G\left(\kappa, \Omega_{s_{1}, d}\right)}{\Omega_{s_{1}, d}},
$$

585
Consequently, the probability of $\kappa=1,2, \ldots, M-1$ can be obtained by considering both of the above cases, yielding

$$
\begin{aligned}
P(\kappa)= & P_{1}(\kappa)+P_{2}(\kappa) \\
= & \frac{\Lambda}{1+\Lambda}\left[\frac{\left(2 / \Omega_{S_{1}, \delta}\right)+2 G\left(0.5, \Omega_{S_{1}, \delta}\right)-2}{(M-1)}\right. \\
& \left.+\frac{(M-\kappa+1) G\left(\kappa, \Omega_{s_{1}, d}\right)}{\Omega_{s_{1}, d}}\right],
\end{aligned}
$$

for $\kappa=1,2, \ldots, M-1$.

3.3 $\kappa=M$

As shown in Fig. 3, when the FD state $S_{1}$ with a duration of $\delta \geq 2$ occurs, we have $\kappa=M$ for the $j$ th, $j \in\{2, \ldots, \delta-1\}$, OFDM symbols. Therefore, we arrive at

$$
P(\kappa)=\sum_{\delta=2}^{\infty} \frac{\delta-2}{\delta} P\left(\delta, S_{1} \mid \widetilde{\boldsymbol{X}}\right), \quad \kappa=M
$$

Upon substituting (18) into the above equation, we obtain

$$
\begin{aligned}
P(\kappa) & =\frac{\Lambda}{1+\Lambda} \frac{\sum_{\delta=2}^{\infty} \delta p\left(\delta \mid S_{1}\right)-2 \sum_{\delta=2}^{\infty} p\left(\delta \mid S_{1}\right)}{\Omega_{S_{1}, \delta}} \\
& =\frac{\Lambda}{1+\Lambda}\left[2-\frac{2}{\Omega_{S_{1}, \delta}}-G\left(0.5, \Omega_{S_{1}, \delta}\right)\right]
\end{aligned}
$$

for $\kappa=M$.

In summary, the PMF of $\kappa$ is given in the following equation (see (28))

\section{Analysis of the average BER}

In this section, we first analyse the signal-to-noise ratio (SNR) based on our noise model. Then, a closed-form formula is derived for the average BER of the OFDM-modulated PLC system.

\subsection{SNR analysis}

According to (4) and (13), the instantaneous SNR per symbol for the $i$ th subchannel is given by

$$
\begin{aligned}
\gamma_{i, \kappa} & =\frac{\left|H_{i}\right|^{2} E_{\mathrm{s}}}{[1+(\mu-1)(\kappa / M)] \sigma_{s_{0}, n}^{2}} \\
& =\frac{\left|H_{i}\right|^{2}}{1+(\mu-1)(\kappa / M)} \times \gamma_{s_{0}, n},
\end{aligned}
$$

where $H_{i}$ is the fading gain of the $i$ th subchannel, $E_{\mathrm{s}}$ is the signal energy per symbol and, by definition, $\gamma_{s_{0}, n}=E_{\mathrm{s}} \sigma_{s_{0}, n}^{2}$ denotes the SNR encountered in state $s_{0}$. Equation (29) shows that $\gamma_{i, \kappa}$ depends on both the channel fading and on the time-variant noise power. As mentioned in Section 2.2, the PLC channel can be assumed to be time-invariant during an OFDM symbol. Thus, the
595 605 610 615 620 625 630 
Table 1 Parameters $\left[\rho_{l}, \theta_{l}\right]$ for different modems

\begin{tabular}{lcccc}
\hline & BPSK & QPSK & 16OAM & 64 QAM \\
\hline$\left[\rho_{1}, \theta_{1}\right]$ & {$[1, \sqrt{2}]$} & {$[1,1]$} & {$\left[\frac{3}{4}, \sqrt{\frac{1}{5}}\right]$} & {$\left[\frac{7}{12}, \sqrt{\frac{1}{21}}\right]$} \\
{$\left[\rho_{2}, \theta_{2}\right]$} & - & - & {$\left[\frac{1}{2}, 3 \sqrt{\frac{1}{5}}\right]$} & {$\left[\frac{1}{2}, 3 \sqrt{\frac{1}{21}}\right]$} \\
{$\left[\rho_{3}, \theta_{3}\right]$} & - & - & {$\left[\frac{1}{4}, 5 \sqrt{\frac{1}{5}}\right]$} & {$\left[-\frac{1}{12}, 5 \sqrt{\frac{1}{21}}\right]$} \\
{$\left[\rho_{4}, \theta_{4}\right]$} & - & - & & {$\left[\frac{1}{12}, 9 \sqrt{\frac{1}{21}}\right]$} \\
{$\left[\rho_{5}, \theta_{5}\right]$} & - & - & & \\
\hline
\end{tabular}

675

fading gain of all the subchannels is the same during the transmission of an OFDM. However, $\kappa$ is a random variable with the PMF given by (28).

\subsection{Average BER}

Given the SNR, the average BER of the OFDM-assisted PLC system can be obtained by averaging the conditional BER $P_{e}\left(\gamma_{i, \kappa}\right)$ over the distribution of the SNR $\gamma_{i, \kappa}$, which is expressed as

$$
P_{b}=\frac{1}{M} \sum_{i=0}^{M-1} \sum_{\kappa=0}^{M} P_{e}\left(\gamma_{i, \kappa}\right) p(\kappa),
$$

where $p(\kappa)$ is given in (28). In (30), $P_{e}\left(\gamma_{i, k}\right)$ is the BER of $Q$-ary QAM employing Gray coding for a given SNR $\gamma_{i, \kappa}$, which can be expressed in a generalised form as [33, 34]

$$
P_{e}\left(\gamma_{i, \kappa}\right)=\sum_{l} \rho_{l} Q\left(\theta_{l} \sqrt{\gamma_{i, \kappa}}\right)
$$

where $Q(x)=(1 / \sqrt{2 \pi}) \int_{x}^{\infty} \mathrm{e}^{-t^{2} 2} \mathrm{~d} t$ is the Gaussian $Q$-function, while the values of $\rho_{l}$ and $\theta_{l}$ for the different modulation schemes are given in Table 1. Finally, upon substituting (28) and (31) into (30), the average BER of the OFDM-assisted PLC system under our noise model can be shown in the following equation:

$$
\begin{aligned}
& P_{b}=\frac{1}{M(1+\Lambda)} \sum_{i=0}^{M-1}\left\{P_{e}\left(\left|H_{i}\right|^{2} \gamma_{s_{0}, n}\right)+\frac{\Lambda}{\Omega_{s_{1}, d}}\right. \\
& \sum_{\kappa=1}^{M-1}(M-\kappa+1) G\left(\kappa, \Omega_{s_{1}, d}\right) P_{e}\left(\frac{\left|H_{i}\right|^{2}}{1+(\mu-1)(\kappa / M)} \gamma_{s_{0}, n}\right) \\
& +\Lambda\left[\frac{\left(2 / \Omega_{S_{1}, \delta}\right)+2 G\left(0.5, \Omega_{S_{1}, \delta}\right)-2}{(M-1)}\right] \\
& \sum_{\kappa=1}^{M-1} P_{e}\left(\frac{\left|H_{i}\right|^{2}}{1+(\mu-1)(\kappa / M)} \gamma_{s_{0}, n}\right) \\
& \left.+\Lambda\left[2-\frac{2}{\Omega_{S_{1}, \delta}}-G\left(0.5, \Omega_{S_{1}, \delta}\right)\right] P_{e}\left(\frac{\left|H_{i}\right|^{2}}{\mu} \gamma_{s_{0}, n}\right)\right\} .
\end{aligned}
$$

Table 2 4-path channel model [8]

\begin{tabular}{lcccc}
\hline \multicolumn{5}{c}{$\alpha(f)=0.78 f(\mathrm{~ns} \mathrm{~Hz} / \mathrm{m}), v_{p}=2 \times 10^{8} \mathrm{~m} / \mathrm{s}$} \\
\hline$I$ & 1 & 2 & 3 & 4 \\
\hline$g_{i}$ & 0.6400 & 0.3800 & -0.1500 & 0.0500 \\
$\tau_{i}$, s & 1.0000 & 1.1120 & 1.2240 & 1.3375 \\
\hline
\end{tabular}

\section{Performance results}

In this section, the BER performance of OFDM-assisted PLC systems is evaluated by comparing the analytical and simulation results for a bandwidth of $25 \mathrm{MHz}$. A sampling frequency of 50 $\mathrm{MHz}$ is used for meeting the Nyquist criterion, which leads to the chip duration of $20 \mathrm{~ns}$. For the indoor PLC channels, the 4-path model of [8] is employed and the corresponding parameters are given in Table 2, while the corresponding CTF is shown in Fig. 4. In the $\mathrm{FD}$, the correlation coefficient of the CTF can be expressed as

$$
R_{\Delta f}=\frac{E\left[H(f) H^{*}(f+\Delta f)\right]}{E\left[|H(f)|^{2}\right]}
$$

where $H(f)$ is given in (5) and $H^{*}(f)$ denotes the conjugate of $H(f)$. Upon substituting the parameters of Table 2 into (5), the $90 \%$ coherence bandwidth of the channel can be obtained with the aid of (33), which is equal to $588.24 \mathrm{kHz}$. In order to guarantee that each OFDM subchannel exhibits flat fading, the number of subcarriers of the OFDM system should satisfy $M \gg(25 \mathrm{MHz}$ $/ 0.58824 \mathrm{MHz}) \simeq 42.5$. Hence, in our studies, we opted for $M=256$. In addition, in order to avoid the ISI, the length of the CP is chosen to be $L^{\prime}=50$, which leads to $L^{\prime} \Delta t>\tau_{\max }$.

The measurement results of [16] show that the average duration of the IN in PLCs is about $\Omega_{s_{1}, t_{d}}=60 \mu \mathrm{s}$ for typical and weak impairments, which are used in our simulations, while the average inter-arrival time varies from a few seconds to a few milliseconds in practical scenarios. In order to increase the associated flexibility, the ADIR $\Lambda$ defined in Section 2.3 is varied in order to obtain different average inter-arrival times. For a non-dispersive channel we let $L^{\prime}=0$ and $H_{i}=1$ for $i=0,1, \ldots, M-1$.

We infer from the above discussions that in order to mitigate the ISI, the length of CP is chosen to be 50 samples in our simulations, hence the system's normalised transmission rate is reduced to $M(M+$ $\left.L^{\prime}\right) \simeq 0.837$, which results in about $0.77 \mathrm{~dB}$ loss of SNR. Observe from (29) that the SNR is jointly determined by both the CTF and the IN, which is characterised by the parameter $\mu$. Since the CTF is independent of the noise, we may analyse their effects on the system's performance separately.

In Fig. 5, we study the BER performance of the OFDM system considered, when communicating over either non-dispersive additive white Gaussian noise (AWGN) channels or over dispersive PLC channels subjected to both IN and AWGN. In this figure, four different modulation schemes are considered, which are BPSK, QPSK, 16QAM and 64QAM. In addition to the well-understood classic features of the different modulation schemes, from the results of Fig. 5 we infer the following observations. First, when the PLC channels become dispersive, the

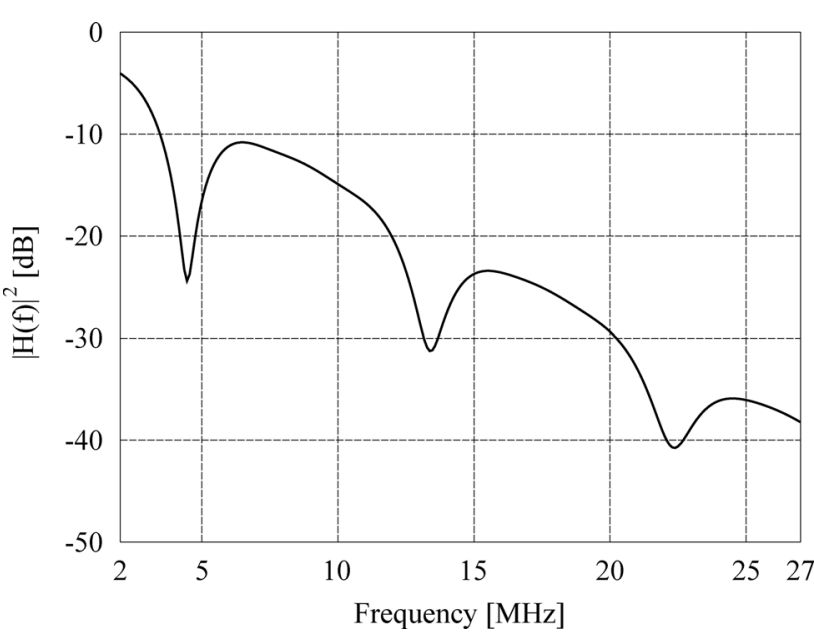

Fig. 4 4-path model of the indoor PLC channel of [8]. Corresponding channel parameters are given in Table 2 


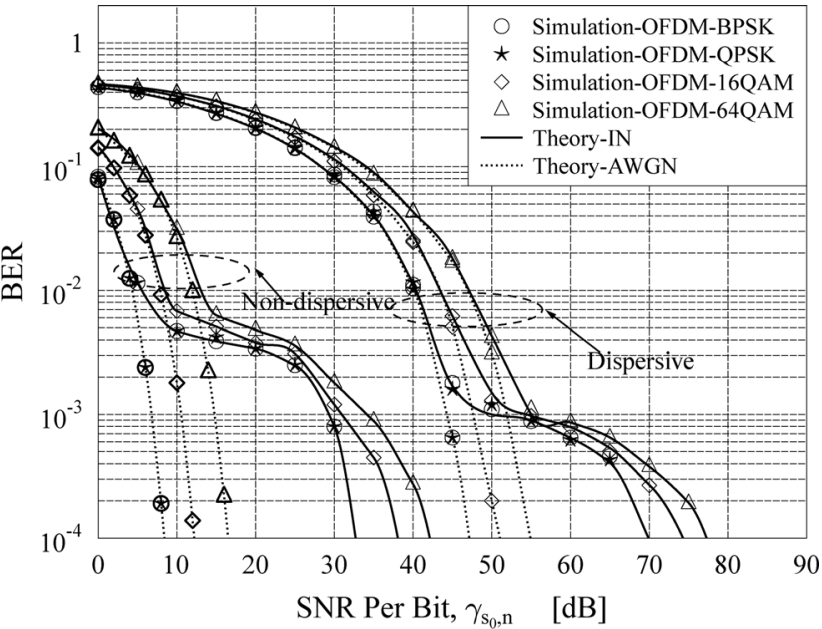

810

Fig. 5 BER performance of OFDM systems associated with $M=256$ and $L^{\prime}=50$ using BPSK, QPSK, 16QAM, or 64QAM when communicating over either non-dispersive AWGN channels or dispersive PLC channels contaminated both by IN and AWGN. Average inter-arrival time of the IN bursts is $\Omega_{s_{0}, t_{d}}=6 \mathrm{~ms}$, the average duration of the IN bursts is

$815 \Omega_{s_{1}, t_{d}}=60 \mu \mathrm{s}$, and the ratio between the IN power and the background noise power is $\mu=30 \mathrm{~dB}$

BER performance significantly degrades. As shown in Fig. 5, for a given BER of $10^{-4}$, communicating over dispersive PLC channels

820 requires as much as $40 \mathrm{~dB}$ higher SNR $\gamma_{s_{0, n}}$ than communicating over non-dispersive AWGN channels. This performance loss may be explained with the aid of Fig. 4, where the deep fades of the channel may reach $40 \mathrm{~dB}$ attenuation, hence the BER is severely degraded. Second, the impulse noise also severely affects the achievable BER performance. As shown in Fig. 5, when the PLC 25 channels experience both IN and AWGN, marked as 'Theory-IN', about $25 \mathrm{~dB}$ of SNR loss is observed in comparison to the PLC channels experiencing only AWGN.

In Fig. 6, we investigate the effect of ADIR on the BER performance of OFDM-based PLC systems having $M=256$

830 subcarriers and a CP length of $L^{\prime}=0$, when communicating over non-dispersive channels subjected to both IN and AWGN. In this figure, only BPSK is considered, but the BER curves of the higher-order $Q$-ary QAM obey similar tendencies. In comparison to the channels experiencing only AWGN, when the channels

835

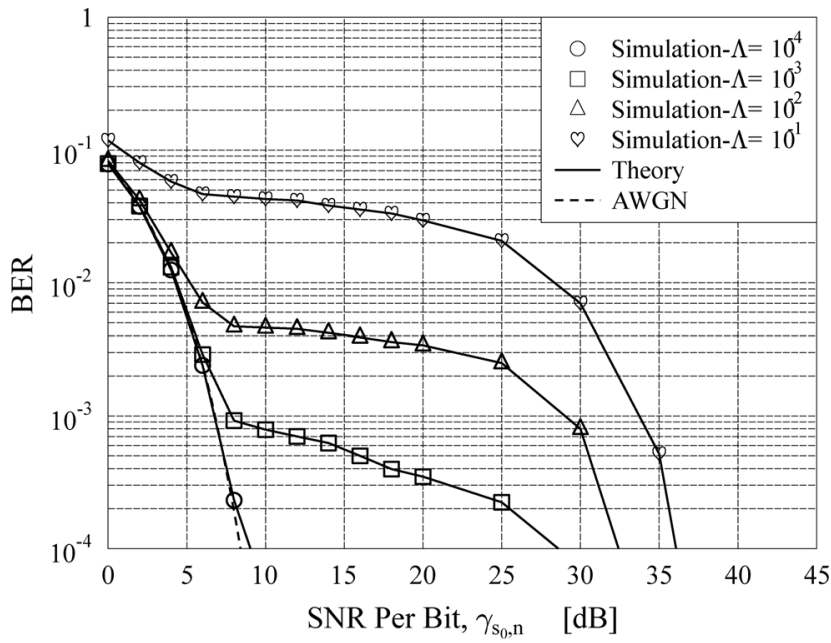

Fig. 6 BER performance of the OFDM system for $M=256$ and $L^{\prime}=0$ using $B P S K$, when communicating over a non-dispersive channel subjected to both $I N$ and $A W G N$. Average duration of bursts is $\Omega_{s_{1}, t_{d}}=60 \mu \mathrm{s}$, while the average inter-arrival time varies with the value of the ADIR $\Lambda$. Ratio between the IN power and the background noise power is $\mu=30 \mathrm{~dB}$

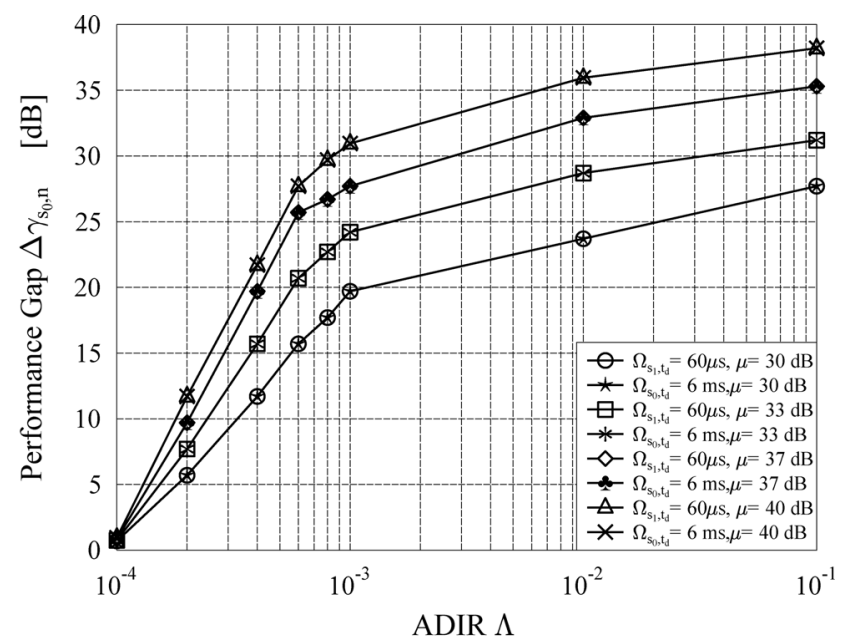

860

865

Fig. 7 Performance gap $\Delta \gamma_{s_{0^{\prime}} \text { n }}$ per bit against the ADIR $\Lambda$ at a BER of $10^{-4}$ OFDM system relies on $M=256$ and $L^{\prime}=0$ using BPSK, when communicating over a non-dispersive channel subjected to both IN and $A W G N$

experience both IN and AWGN, there is a gradual slope change for the BER curve at a certain value of SNR, as shown in Fig. 6. This implies that before this point the system performance is dominated by the AWGN, and then more and more by the IN. As a result of IN, the system's performance is degraded predominantly depending on the value of ADIR. When the ADIR is increased, the system performance degradation becomes more severe, because according to (32), the probability of an OFDM symbol becoming impaired by high-power IN bursts increases.

In Fig. 7, we show the effect of ADIR on the performance gap $\Delta \gamma_{s_{0}}$ between the channels experiencing AWGN alone and the channels experiencing both IN as well as AWGN at a BER of $10^{-4}$. First, according to the definition of the ADIR in Section 2.3, we can change the value of $\Lambda$ either by keeping $\Omega_{s_{1}, t_{d}}$ constant and changing $\Omega_{s_{0}, t_{d}}$, or by keeping $\Omega_{s_{0}, t_{d}}$ constant, but varying $\Omega_{s_{1}, t_{d}}$. As shown in Fig. 7 , when $\Lambda$ varies from $10^{-4}$ to $10^{-1}$, the performance results associated with keeping $\Omega_{s_{1}, t_{d}}$ constant are the same as those of keeping $\Omega_{s_{0}, t_{d}}$ constant. Second, observe in Fig. 7 that when the ADIR increases, the performance gap gradually increases, but asymptotically tending to a certain value. This observation can be explained with the aid of (32), which shows that as the value of $\Lambda$ becomes larger, the performance becomes similar to that of the system, where the channels experience only AWGN with a variance of $\mu \sigma_{s_{0}, n}^{2}$.

\section{Conclusions}

In this paper, a HSMM was invoked for representing the statistical properties of the asynchronous IN encountered in PLC channels. A closed-form BER expression has been derived for the OFDM system communicating over dispersive indoor PLC channels experiencing both background and asynchronous IN. The accuracy of our analytical results has been verified by our simulation results. Furthermore, the BER performance has been investigated for different scenarios both numerically and by simulations. We have shown that the system performance is severely degraded by the CTF of PLC channels, which is aggravated by the asynchronous IN. Therefore, the effect of asynchronous IN has to be mitigated by efficient techniques, which will be the subject of our future research.

\section{Acknowledgments}

The financial support of the European Research Council's (ERC) Advanced Fellow Grant was gratefully acknowledged. 
1 Hrasnica, H., Haidine, A., Lehnert, R.: 'Broadband powerline communications: network design' (Wiley, 2005)

2 Galli, S., Scaglione, A., Wang, Z.: 'For the grid and through the grid: The role of power line communications in the smart grid', Proc. IEEE, 2011, 99, (6), pp. $998-1027$

3 Ma, Y.H., So, P., Gunawan, E.: 'Performance analysis of OFDM systems for broadband power line communications under impulsive noise and multipath effects', IEEE Trans. Power Deliv., 2005, 20, (2), pp. 674-682

4 'IEEE Standard for Broadband over Power Line Networks: Medium Access Control and Physical Layer Specifications', IEEE Std 1901-2010, 2010, pp. 1-1586

HomePlug AV2 White Paper. HomePlug Powerline Alliance. Available as: http://www.homeplug.org/

6 I.-T. R. G.9960: 'Unified high-speed wireline-based home networking transceivers-system architecture and physical layer specification', 2011

7 Ferreira, H.C., Lampe, L., Newbury, J., Swart, T.G. (Eds.): 'Power line communications: theory and applications for narrowband and broadband communications over power lines' (Wiley-Blackwell, 2010, 1st edn.)

8 Zimmermann, M., Dostert, K.: 'A multipath model for the powerline channel', IEEE Trans. Commun., 2002, 50, (4), pp. 553-559

9 Anastasiadou, D., Antonakopoulos, T.: 'Multipath characterization of indoor power-line networks', IEEE Trans. Power Deliv., 2005, 20, (1), pp. 90-99

10 Galli, S., Banwell, T.: 'A deterministic frequency-domain model for the indoor power line transfer function', IEEE J. Sel. Areas Commun., 2006, 24, (7), pp. $1304-1316$

11 Tlich, M., Zeddam, A., Moulin, F., et al.: 'Indoor power-line communications channel characterization up to $100 \mathrm{MHz}$ - Part II: Time-frequency analysis', IEEE Trans. Power Deliv., 2008, 23, (3), pp. 1402-1409

12 Galli, S.: 'A simplified model for the indoor power line channel'. IEEE Int. Symp. on Power Line Communications and its Applications, 2009, ISPLC 2009, March 2009, pp. 13-19

3 Tonello, A., Versolatto, F.: 'Bottom-up statistical PLC channel modeling - Part II: Inferring the statistics', IEEE Trans. Power Deliv., 2010, 25, (4), pp. 2356-2363

14 Galli, S.: 'A novel approach to the statistical modeling of wireline channels', IEEE Trans. Commun., 2011, 59, (5), pp. 1332-1345

15 Tonello, A., Versolatto, F., Béjar, B., et al.: 'A fitting algorithm for random modeling the PLC channel', IEEE Trans. Power Deliv., 2012, 27, (3), pp. $1477-1484$

Zimmermann, M., Dostert, K.: 'Analysis and modeling of impulsive noise in broad-band powerline communications', IEEE Trans. Electromagn. Compat., 2002, 44, (1), pp. 249-258

17 Meng, H., Guan, Y., Chen, S.: 'Modeling and analysis of noise effects on broadband power-line communications', IEEE Trans. Power Deliv., 2005, 20, (2), pp. 630-637

18 Channel Model Sub-committee of P1901: 'Electrical network and topology channel and noise model'. P1901-10-0356-00, Tech. Rep., September 2010

19 FP7 Theme 3 ICT-213311 OMEGA: 'PLC channel characteriztion and modelling'. Tech. Rep., December 2008

20 Blackard, K., Rappaport, T., Bostian, C.: 'Measurements and models of radio frequency impulsive noise for indoor wireless communications', IEEE J. Sel. Areas Commun., 1993, 11, (7), pp. 991-1001

21 Kassam, S.A.: 'Signal detection in non-Gaussian noise' (Springer-Verlag, 1988)

22 Ghosh, M.: 'Analysis of the effect of impulse noise on multicarrier and single carrier QAM systems', IEEE Trans. Commun., 1996, 44, (2), pp. 145-147

23 Middleton, D.: 'Non-Gaussian noise models in signal processing for telecommunications: new methods and results for class A and class B noise models', IEEE Trans. Inf. Theory, 1999, 45, (4), pp. 1129-1149

24 Amirshahi, P., Navidpour, S., Kavehrad, M.: 'Performance analysis of uncoded and coded OFDM broadband transmission over low voltage power-line channels with impulsive noise', IEEE Trans. Power Deliv., 2006, 21, (4), pp. 1927-1934

25 Fertonani, D., Colavolpe, G.: 'On reliable communications over channels impaired by bursty impulse noise', IEEE Trans. Commun. 2009, 57, (7), pp. 2024-2030

26 Ndo, G., Labeau, F., Kassouf, M.: 'A Markov-Middleton model for bursty impulsive noise: modeling and receiver design', IEEE Trans. Power Deliv., 2013, 28, (4), pp. 2317-2325

27 Levinson, S.E.: 'Continuously variable duration hidden Markov models for automatic speech recognition', Comput. Speech Lang., 1986, 1, (1), pp. 29-45

28 Yu, S.-Z.: 'Hidden semi-Markov models', Artif. Intell, 2009, 174, (2), pp. 215-243

29 Yang, L.-L.: 'Multicarrier communications' (John Wiley \& Sons, Ltd, 2009)

30 Hanzo, L., Ng, S.X., Webb, W.T., et al.: 'Quadrature amplitude modulation: from basics to adaptive trellis-coded, turbo-equalised and space-time coded OFDM, CDMA and MC-CDMA systems' (IEEE Press-John Wiley, 2004)

31 Rabiner, L.: 'A tutorial on hidden Markov models and selected applications in speech recognition', Proc. IEEE, 1989, 77, (2), pp. 257-286

32 Bertsekas, D., Gallager, R.: 'Data networks' (Prentice-Hall, 1992)

33 Yang, L.-L., Hanzo, L.: 'A recursive algorithm for the error probability evaluation of M-QAM', IEEE Commun. Lett., 2000, 4, (10), pp. 304-306

34 Vitthaladevuni, P., Alouini, M.-S.: 'A recursive algorithm for the exact BER computation of generalized hierarchical QAM constellations', IEEE Trans. Inf. Theory, 2003, 49, (1), pp. 297-307

985435 Zimmermann, M., Dostert, K.: 'An analysis of the broadband noise scenario in powerline networks'. Proc. ISPLC, April 2000, pp. 131-138

36 Abramowitz, M., Stegun, I.A. (Eds.): 'Handbook of mathematical functions with formulas, graphs, and mathematical tables' (Dover Publications, New York, 1972)

37 Chariag, D., Guezgouz, D., Le Bunetel, J.C., et al.: 'Modeling and simulation of temporal variation of channel and noise in indoor power-line network', IEEE Trans. Power Deliv., 2012, 27, (4), pp. 1800-1808

\section{Appendices: derivation of $p\left(d \mid s_{i}\right)$ and $p\left(\delta \mid S_{i}\right)$}

This Appendix derives the PMFs of $d$ conditioned on $s_{0}$ and $s_{1}$, as well as the PMFs of $\delta$ conditioned on $S_{0}$ and $S_{1}$.

First, it can be shown that for a given state $s_{i}$, where $i=0,1$, we 995

$$
\begin{aligned}
p\left(d \mid s_{i}\right) & =\int_{(d-0.5) \Delta t}^{(d+0.5) \Delta t} \frac{1}{\Omega_{s_{i}, t_{d}}} \exp \left(-\frac{t_{d}}{\Omega_{s_{i}, t_{d}}}\right) \mathrm{d} t_{d} \\
& =\exp \left(-\frac{(d-0.5) \Delta t}{\Omega_{s_{i}, t_{d}}}\right)-\exp \left(-\frac{(d+0.5) \Delta t}{\Omega_{s_{i}, t_{d}}}\right) \\
& =\exp \left(-\frac{d-0.5}{\Omega_{s_{i}, d}}\right)-\exp \left(-\frac{d+0.5}{\Omega_{s_{i}, d}}\right) .
\end{aligned}
$$

Then, according to (8), we can show that

$$
\begin{aligned}
\sum_{d=x_{1}}^{x_{2}} p\left(d \mid s_{i}\right)= & \exp \left(-\frac{x_{1}-0.5}{\Omega_{s_{i}, d}}\right)-\exp \left(-\frac{x_{2}+0.5}{\Omega_{s_{i}, d}}\right) \\
\sum_{d=x_{1}}^{x_{2}} d p\left(d \mid s_{i}\right)= & \Omega_{s_{i}, d} \exp \left(-\frac{x_{1}+0.5}{\Omega_{s_{i}, d}}\right) \\
& -\Omega_{s_{i}, d} \exp \left(-\frac{x_{2}-0.5}{\Omega_{s_{i}, d}}\right) \\
& +x_{1} \exp \left(-\frac{x_{1}-0.5}{\Omega_{s_{i}, d}}\right)-x_{2} \exp \left(-\frac{x_{2}+0.5}{\Omega_{s_{i}, d}}\right) ;
\end{aligned}
$$

For the state $S_{0}$, we can rewrite (11) as

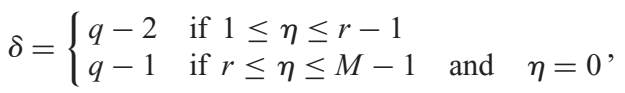

where we have $q=\lceil d M\rceil$ and $r=\lceil d M\rceil M-d$, while $\lceil x\rceil$ denotes the smallest integer not smaller than $x$. Since $d$ and $\eta$ are independent, with the aid of (8) and (10), we have

$$
\begin{aligned}
p\left(\delta \mid S_{0}\right)= & \sum_{d=(\delta+1) M}^{(\delta+2) M} \frac{(\delta+2) M-d-1}{M} p\left(d \mid s_{0}\right) \\
& +\sum_{d=\delta M}^{(\delta+1) M} \frac{d-\delta M+1}{M} p\left(d \mid s_{0}\right) \\
\simeq & \frac{\Omega_{s_{0}, d}+1}{M}\left[\exp \left(-\frac{\delta M}{\Omega_{s_{0}, d}}\right)-2 \exp \left(-\frac{(\delta+1) M}{\Omega_{s_{0}, d}}\right)\right. \\
& \left.+\exp \left(-\frac{(\delta+2) M}{\Omega_{s_{0}, d}}\right)\right]
\end{aligned}
$$

where the mean of $\delta$ conditioned on $S_{0}$ can be calculated as

$$
\Omega_{S_{0}, \delta}=\sum_{\delta=1}^{\infty} \delta p\left(\delta \mid S_{0}\right)=\frac{\Omega_{s_{0}, d}+1}{M} \exp \left(-\frac{M}{\Omega_{s_{0}, d}}\right) .
$$

In practice, we usually have $\Omega_{s_{0}, t_{d}} \gg T_{\mathrm{s}}$. As a result, we have $\Omega_{s_{0}, d} \gg M$. Thus, we may exploit the following approximation

$$
\Omega_{S_{0}, \delta} \simeq \frac{\Omega_{s_{0}, d}+1}{M} \simeq \frac{\Omega_{s_{0}, d}}{M} .
$$


Consequently, when substituting (40) into (38), we obtain

$$
p\left(\delta \mid S_{0}\right) \simeq \Omega_{S_{0}, \delta}
$$

1060

$$
\left[\exp \left(-\frac{\delta}{\Omega_{S_{0}, \delta}}\right)-2 \exp \left(-\frac{\delta+1}{\Omega_{S_{0}, \delta}}\right)+\exp \left(-\frac{\delta+2}{\Omega_{S_{0}, \delta}}\right)\right] .
$$
1065

For the state $S_{1}$, we can rewrite (11) as

$$
\delta=\left\{\begin{array}{ll}
q & \text { if } 0 \leq \eta \leq r \\
q+1 & \text { if } r+1 \leq \eta \leq M-1
\end{array},\right.
$$

where the variables have the same meaning as those in (37). 1070

Similarly to (38), with the aid of (8) and (10), we have

$$
\begin{aligned}
p\left(\delta \mid S_{1}\right) & =\sum_{d=0}^{M} \frac{M-d+1}{M} p\left(d \mid s_{1}\right) \\
& \simeq \frac{\Omega_{s_{1}, d}-1}{M} \exp \left(-\frac{M}{\Omega_{s_{1}, d}}\right), \quad \delta=1,
\end{aligned}
$$

and

1080

1085

1090

$$
\begin{aligned}
p\left(\delta \mid S_{1}\right)= & \sum_{d=(\delta-1) M}^{\delta M} \frac{\delta M-d+1}{M} p\left(d \mid s_{1}\right) \\
& +\sum_{d=(\delta-2) M}^{(\delta-1) M} \frac{d-(\delta-2) M-1}{M} p\left(d \mid s_{1}\right) \\
\simeq & \frac{\Omega_{s_{1}, d}-1}{M}\left[\exp \left(-\frac{(\delta-2) M}{\Omega_{s_{1}, d}}\right)\right. \\
& -2 \exp \left(-\frac{(\delta-1) M}{\Omega_{s_{1}, d}}\right) \\
& \left.+\exp \left(-\frac{\delta M}{\Omega_{s_{1}, d}}\right)\right], \quad \delta=2,3, \ldots
\end{aligned}
$$

1095

Similar to (39) and (40), the mean of $\delta$ conditioned on $S_{1}$ can be calculated as

$$
\Omega_{S_{1}, \delta}=\sum_{\delta=1}^{\infty} \delta p\left(\delta \mid S_{1}\right) \simeq \frac{\Omega_{s_{1}, d}}{M}
$$

In addition, the relationship between $\Omega_{S_{1, \delta}}$ and $\Omega_{S_{0}, \delta}$ can be expressed as

$$
\frac{\Omega_{S_{1}, \delta}}{\Omega_{S_{0}, \delta}}=\frac{\Omega_{s_{1}, d}}{\Omega_{s_{0}, d}}=\Lambda
$$

Then, upon substituting (44) into (43a) and (43b), gives

$$
p\left(\delta \mid S_{1}\right) \simeq \Omega_{S_{1}, \delta} \exp \left(-\frac{1}{\Omega_{S_{1}, \delta}}\right), \quad \delta=1,
$$

and

$$
\begin{aligned}
p\left(\delta \mid S_{1}\right) \simeq & \Omega_{S_{1}, \delta}\left[\exp \left(-\frac{\delta-2}{\Omega_{S_{1}, \delta}}\right)-2 \exp \left(-\frac{\delta-1}{\Omega_{S_{1}, \delta}}\right)\right. \\
& \left.+\exp \left(-\frac{\delta}{\Omega_{S_{1}, \delta}}\right)\right], \quad \delta=2,3, \ldots
\end{aligned}
$$

Furthermore, let us define

1145

$$
G(x, \Omega)=\exp \left(-\frac{x-0.5}{\Omega}\right)-\exp \left(-\frac{x+0.5}{\Omega}\right)
$$

Then, (46b) can be rewritten as

$$
\begin{gathered}
p\left(d \mid s_{i}\right)=G\left(d, \Omega_{s_{i}, d}\right) \\
p\left(\delta \mid S_{i}\right)=\Omega_{S_{i}, \delta}\left[G\left(\frac{\delta+(-1)^{i}}{2}, \Omega_{S_{i}, \delta}\right)\right]^{2}-\zeta\left(\Omega_{S_{i}, \delta}\right),
\end{gathered}
$$

where

$$
\zeta\left(\Omega_{S_{i}, \delta}\right)= \begin{cases}\Omega_{S_{i}, \delta}\left[\mathrm{e}^{\left(1 / \Omega_{S_{i}, \delta}\right)}-2\right] & \text { if } \delta=1 \text { and } i=1 \\ 0 & \text { otherwise }\end{cases}
$$


Author Queries

Hongming Zhang, Lie-Liang Yang, Lajos Hanzo

Q1 Please confirm the changes made in the article title.

Q2 Please check the email address of the corresponding author.

Q3 Footnote has been moved to text as required by journal style. Please check and confirm that it has been located correctly within the text.

Q4 References [35-37] are listed in the reference list but not cited in the text. Please cite in the text, else delete from the list. 